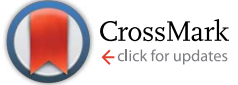

Cite this: Anal. Methods, 2015, 7, 9663

\title{
A fluorophore-conjugated ascorbic acid functions for the visualization of sodium vitamin C transporters in living cells
}

\begin{abstract}
Junfa Yin, ${ }^{a}$ Yuanyuan Song, ${ }^{a}$ Ning Zhang, ${ }^{\text {ab }}$ Tian $\mathrm{Xu}^{\mathrm{a}}$ and Hailin Wang*a
Human beings do not produce ascorbic acid (AA) and acquire AA through the sodium vitamin $C$ transporters (SVCTs). Changes in the expression or function of SVCT proteins may be associated with human diseases. In this regard, imaging of SVCTs in living cells is important. However, the options for live-cell labelling of SVCTS were very limited. In this work, we synthesized a new small-molecule fluorescent probe RB-A-Vc, and demonstrated its application in selectively visualizing SVCTs in living cells. This probe features visible excitation and emission profiles, can easily enter into membranes, has high selectivity for SVCTs, and can monitor up-regulation or down-regulation of SVCT expression in living cells. We emphasize that this smallmolecule probe is suitable for subcellular localization of SVCTs in living cells. This study provides a useful tool for simultaneously monitoring the level and distribution of intracellular SVCTs, which is probably more useful for evaluating the changes induced by external stimulations. We propose that this probe for SVCT imaging and the corresponding method could be applied to other cell lines, tissues, and species.
\end{abstract}

Received 19th July 2015

Accepted 30th September 2015

DOI: 10.1039/c5ay01883h

www.rsc.org/methods

distribution and spatiotemporal dynamics. Up to now, the options for live cell labelling of SVCTs were very limited. The antibodies against SVCT1 and/or SVCT2 in immunofluorescence assays are not membrane permeable. Thus they can only be used for indicating SVCTs in fixed and permeabilized cells. ${ }^{19,20}$ Another important issue is, although the antibodies for SVCT1 and SVCT2 are known, there is a lack of a reporter for monitoring the global level and changes of SVCTs. In fact, previous studies revealed that the biological and toxicological functions of AA transporters are commonly associated with both SVCT1 and SVCT2..,2,9 To the best of our knowledge, to date, there are no fluorescent probes capable of selective detection of the total SVCTs in living cells. The development of easy-to-use probes, which can permeate the cell membrane and selectively bind and illuminate the total SVCTs, was thus required.

Herein, we synthesized a fluorophore-conjugated vitamin C (RB-A-Vc), and demonstrated its application in selectively visualizing SVCTs in living cells. The unique merits of this study include, (1) the synthesized small-molecule compound can easily pass through the cell membrane by binding to SVCTs and thus it is suitable for living cell imaging, and (2) being a potent tool for simultaneously visualizing the level and changes of the total intracellular SVCTs.

\section{Experimental}

${ }^{a}$ State Key Laboratory of Environmental Chemistry and Ecotoxicology, Research Center for Eco-Environmental Sciences, Chinese Academy of Sciences, Beijing 100085, People's Republic of China. E-mail: hlwang@rcees.ac.cn; Tel: +86-10-62849600

${ }^{b}$ Shanghai Key Lab of Functional Materials Chemistry, East China University of Science and Technology, Shanghai 200237, China

\section{Reagents and materials}

L-Ascorbic acid (AA), phorbol 12-myristate 13-acetate (PMA), Triton X-100, Tween 20 and 4',6-diamidino-2-phenylindole 
(DAPI) were purchased from Sigma-Aldrich (MO, USA). Sulforhodamine $\mathrm{B}$ chloride and 1,4-diethylene dioxide were purchased from Acros (NJ, USA). 4-(Dimethylamino)pyridine (DMAP) and paraformaldehyde were purchased from Alfa Aesar. 6-Aminoheanoic acid was purchased from Sangon Biotech (Shanghai, China). $\mathrm{CaCl}_{2} \cdot 2 \mathrm{H}_{2} \mathrm{O}, \mathrm{NaCl}, \mathrm{KCl}, \mathrm{Na}_{2} \mathrm{HPO}_{4}, \mathrm{NaHCO}_{3}$ and $\mathrm{KH}_{2} \mathrm{PO}_{4}$ were purchased from Sinopharm Chemical Reagent Co, Ltd (Beijing, China). Choline chloride was purchased from J\&K Co, Ltd (Beijing, China). Mito-Tracker Green and Dio were purchased from Beyotime Biotechnology (Shanghai, China).

\section{Synthesis of sulforhodamine B-aminoheanoic acid-ascorbic acid ester (RB-A-Vc)}

A mixture of 6-aminoheanoic acid (655 mg) and L-ascorbic acid (704 mg) was stirred in $98 \% \mathrm{H}_{2} \mathrm{SO}_{4}(20 \mathrm{~mL})$ for $24 \mathrm{~h}$ under nitrogen protection. The solution was diluted and neutralized to neutral and the residue was purified by solid phase extraction to give the product $1.40 \mathrm{mg}$ of sulforhodamine B chloride was added to a solution of 1 (29 $\mathrm{mg}$ ) and DMAP (5 mg) in 1,4diethylene dioxide-saturated aqueous sodium bicarbonate (500 $\mu \mathrm{L}: 500 \mu \mathrm{L}$ ), and the reaction mixture was stirred at room temperature for $2 \mathrm{~h}$. The residues were purified by silica gel column chromatography using trichloromethane/methanol ( $4: 1, \mathrm{v} / \mathrm{v})$ as the eluent to give the desired product $2(\mathrm{RB}-\mathrm{A}-\mathrm{Vc})$. After rotary evaporation, the product was lyophilisated to solid and stored at $-20{ }^{\circ} \mathrm{C}$.

\section{UHPLC-ESI-MS/MS analysis of RB-A-Vc}

Sulforhodamine B-aminoheanoic acid-ascorbic acid ester (RBA-Vc) was identified by reverse-phase UHPLC (Agilent 1290 UHPLC system) using a Zorbax Eclipse Plus C 18 column (2.1 mm i.d. $\times 50 \mathrm{~mm}, 1.8 \mu \mathrm{m}$, Agilent Technologies, Palo Alto, CA) with a 6530 quadrupole tandem time of flight mass spectrometer (Agilent Technologies, Palo Alto, CA). The optimized mobile phase consists of $0.1 \% \mathrm{HCOOH}$ (solvent A) and methanol (solvent B). The elution was $50 \%$ of $\mathrm{B}$. The flow rate was set at $0.25 \mathrm{~mL} \mathrm{~min}^{-1}$, the injection volume was $2 \mu \mathrm{L}$, and the column temperature was set at $25{ }^{\circ} \mathrm{C}$. The mass spectrometer was operated in the positive ion mode. Nitrogen was used for nebulization and desolvation. The nebulization gas was set at $35 \mathrm{psi}$, the flow-rate of desolvation gas was $6 \mathrm{~L} \mathrm{~min}^{-1}$, and the source temperature was set at $200{ }^{\circ} \mathrm{C}$. The sheath gas temperature was set at $300{ }^{\circ} \mathrm{C}$ and the flow-rate was $10 \mathrm{~L} \mathrm{~min}^{-1}$. The capillary voltage was set at $3500 \mathrm{~V}$, and the nozzle voltage at $0 \mathrm{~V}$. High purity nitrogen (99.999\%) was used as the collision gas.

\section{Cell culture}

The human-derived hepatic epithelial HepG2 cells and the human cell line HEK-293T (supplied by cell culture centre, Institute of Basic Medical Sciences, Chinese Academy of Medical Sciences, Beijing, China) were grown in RPMI 1640 medium and Dulbecco's modified Eagle's medium (DMEM)high glucose (Hyclone, CE Healthcare Life Sciences) respectively supplemented by $10 \%$ foetal bovine serum (FBS, Gibco® Life technologies), 1\% penicillin and streptomycin (Corning). The cells were cultured at $37{ }^{\circ} \mathrm{C}$ in a $5 \% \quad \mathrm{CO}_{2}$ humidified atmosphere.

\section{Confocal fluorescent imaging of SVCTs in fixed cells}

HepG2 cells and HEK-293T cells were grown on 8-chamber glass-bottom dishes for $24 \mathrm{~h}$, and then fixed in ice-cold $4 \%$ paraformaldehyde for $10 \mathrm{~min}$ and permeabilized with $0.1 \%$ Triton X-100 for $5 \mathrm{~min}$. Then the cells were respectively cultured in an RPMI 1640 and DMEM medium containing RB-A-Vc for $30 \mathrm{~min}$. The cells were counterstained for $10 \mathrm{~min}$ with DAPI to label the nuclei, Mito-tracker green to label the mitochondria and Dio to label the plasma membrane, and then they were analysed by confocal fluorescence microscopy.

\section{Confocal fluorescent imaging of SVCTs in living cells}

The HEK-293T and HepG2 cells were seeded on 8-chamber glass-bottom dishes and incubated for one day. The cells were cultured in an RPMI 1640 and DMEM medium containing RBA-Vc $(100 \mu \mathrm{M})$ for $30 \mathrm{~min}$, respectively. The intracellular SVCTs can then be visualized by confocal fluorescence microscopy directly.

For the SVCT localization investigations, the cells needed to be fixed in $4 \%$ paraformaldehyde and permeabilized with $0.1 \%$ Triton X-100, counterstained with DAPI to label the nuclei, Mito-tracker green to label the mitochondria and Dio to label the plasma membrane.

To evaluate the dosage effect of RB-A-VC on SVCT imaging, the cultured cells were respectively incubated in an RPMI 1640 and DMEM medium containing RB-A-Vc $(10 \mu \mathrm{M}, 20 \mu \mathrm{M}, 100$ $\mu \mathrm{M}, 200 \mu \mathrm{M})$ for $30 \mathrm{~min}$. Then the medium was removed and the cells were counterstained with DAPI, followed by the observation by confocal fluorescence microscopy.

\section{Immunocolocalization of SVCTs in HEK-293T and HepG2 cells}

For confocal fluorescence and colocalization experiments, the HEK-293T and HepG2 cells were cultured in an RPMI 1640 and DMEM medium containing RB-A-Vc $(100 \mu \mathrm{M})$ for $30 \mathrm{~min}$, respectively. The stained cells were fixed in $4 \%$ paraformaldehyde for $10 \mathrm{~min}$ on ice, washed with BSS buffer (150 $\mathrm{mM} \mathrm{NaCl}, 5 \mathrm{mM} \mathrm{KCl}, 1.9 \mathrm{mM} \mathrm{KH} \mathrm{PO}_{4}, 1.1 \mathrm{mM} \mathrm{Na}_{2} \mathrm{HPO}_{4}, 0.3$ $\mathrm{mM} \mathrm{MgSO}_{4} \cdot 7 \mathrm{H}_{2} \mathrm{O}, 1 \mathrm{mM} \mathrm{MgCl}{ }_{2} \cdot 6 \mathrm{H}_{2} \mathrm{O}, 1.5 \mathrm{mM} \mathrm{CaCl}_{2} \cdot 2 \mathrm{H}_{2} \mathrm{O}, 10$ mM HEPES, adjusted to $\mathrm{pH} 7.6$ with $\mathrm{NaOH}$ ), and permeabilized with $0.1 \%$ Triton X-100 $(200 \mu \mathrm{L}$ of $10 \%$ Triton-100 was diluted with $19.8 \mathrm{~mL}$ of BSS buffer) for $5 \mathrm{~min}$. Afterwards, the cells were blocked with $5 \%$ non-fat dry milk in TBST $(10 \mathrm{~mL}$ Tris-HCl $\mathrm{pH}$ 7.5, $8.8 \mathrm{~g} \mathrm{NaCl}, 1 \mathrm{~mL}$ Tween-20 in $1 \mathrm{~L}$ water) for $30 \mathrm{~min}$, incubated for $3 \mathrm{~h}$ with primary antibodies: anti-SVCT1 (sc-9923 polyclonal, goat), anti-SVCT2 (sc-9926 goat polyclonal), antiCOXIV (ab-16056, rabbit polyclonal) and anti-GLUT1 (ab-40084 mouse monoclonal), washed with BSS buffer 3 times and incubated for $1 \mathrm{~h}$ with fluorescent secondary antibodies: fluorescein-conjugated affiniPure Donkey Anti-Mouse IgG $(\mathrm{H}+\mathrm{L})$ (ab10081) (in green fluorescence), fluorescein-conjugated affiniPure Donkey Anti-Rabbit IgG (H+L) (ab10051) (in green fluorescence), Cy3-conjugated affiniPure Donkey Anti-Goat IgG 
$(\mathrm{H}+\mathrm{L})(\mathrm{ab} 10112)$ (in red fluorescence), fluorescein-conjugated affiniPure Donkey Anti-Goat IgG (H+L) (00003-3) (in green fluorescence).

\section{Cytoplasmic extraction and Western blot}

Protease inhibitors (1:100 dilution) and DL-dithiothreitol (DTT) (1: 1000 dilution) were added to CER I (Thermo Scientific) before use to maintain extract integrity and prevent oxidation. The adherent cells were harvested with trypsin-EDTA (Hyclone CE Healthcare Life Sciences) and centrifuged at $500 \times g$ for 5 minutes. After washing with the BSS buffer, the cells were transferred to a $1.5 \mathrm{~mL}$ microcentrifuge tube and centrifuged at $500 \times g$ for 3 minutes. The supernatant was carefully removed and discarded, leaving the cell pellet as dry as possible. After adding ice-cold CER I $(100 \mu \mathrm{L})$ to the cell pellet, the tube was suspended for 15 seconds and incubated on ice for 10 minutes. Then the tube was added to ice-cold CER II $(5.5 \mu \mathrm{L})$ and incubated for 1 minute. At last the tube was centrifuged for 5 minutes at maximum speed in a microcentrifuge $(16000 \times g)$ and the supernatant (cytoplasmic extract) was transferred to a clean pre-chilled tube. Protein concentrations were measured using the Bradford assay.

$30 \mu \mathrm{g}$ of cytoplasmic proteins was subjected to SDS-PAGE on a $10 \%$ polyacrylamide gel and then electroblotted onto a nitrocellulose membrane. The blotted PVDF membrane was blocked in freshly prepared 5\%-nonfat dry milk for $1 \mathrm{~h}$ at room temperature with constant agitation, then incubated with antiSVCT2 (sc-9926), anti-SVCT1 (sc-9923) and $\beta$-tubulin primary antibodies ( $1: 200$ dilution) (sc-9935) for $2 \mathrm{~h}$ with agitation at room temperature. After washing with TBST three times the membrane was incubated with the Donkey Anti-Goat secondary antibody ( 1 : 2000 dilution) in $5 \%$ milk for 40 min with agitation at room temperature before visualization.

\section{SiRNA transfection}

For siRNA transfection, the cells were transfected with an optimized concentration (7.5 pmol for fluorescence, $35 \mathrm{pmol}$ for Western blot) of SVCT2 siRNA and Lipofectamine 2000 Reagent mixture. Oligonucleotides were diluted in an Opti-MEM (Invitrogen) medium and added to the transfection reagent solution. The cells were incubated in this final mixture for $6 \mathrm{~h}$ at $37^{\circ} \mathrm{C}$. Afterward DMEM, with 10\% fetal bovine serum and antibiotics, was added to the cells and incubated for $72 \mathrm{~h}$ at $37^{\circ} \mathrm{C}$. Then the cells were added into our product for $30 \mathrm{~min}$, washed by PBS 2 times and then stained with DAPI for $5 \mathrm{~min}$.

\section{Imaging/analysis of SVCT expression in cells}

After transfection of siRNA in HEK-293T and HepG2 cells for $72 \mathrm{~h}$ or adding PMA (100 nM) for $12 \mathrm{~h}$, the cells were cultured in an RPMI 1640 and DMEM medium containing RB-A-Vc $(100 \mu \mathrm{M})$ for $30 \mathrm{~min}$. Then the medium was removed, the cells were counterstained with DAPI after they were fixed in ice-cold $4 \%$ paraformaldehyde and analyzed by confocal fluorescence microscopy. The efficiencies of siRNA-depression and PMApromotion of SVCT expression was evaluated by fluorescence spectrometry (excitation at $560 \mathrm{~nm}$, emission at $600 \mathrm{~nm}$ ) using a spectral scanning multimode reader.

\section{Results and discussion}

\section{Synthesis and characterization of RB-A-Vc}

To synthesize such a fluorophore-conjugated vitamin $\mathrm{C}$ for SVCT imaging, our strategy is to introduce fluorescent Rhodamine $\mathrm{B}(\mathrm{RB})$ derivatives to the C-6 hydroxy group of ascorbic acid. Two C-6 substituted esters, Rhodamine B-ascorbic acid ester (RB-Vc) and sulforhodamine B-aminoheanoic acidascorbic acid ester (RB-A-Vc) were synthesized (Fig. 1, Scheme 1). It was found that $\mathrm{RB}-\mathrm{Vc}$ derived by directly conjugating $\mathrm{RB}$ to AA is liable to hydrolyse. The hydrolysed $\mathrm{RB}$ dye is positively charged, can pass through the cell membrane and brings undesired fluorescence, raising the background and disturbing the observation of the probe/SVCT complexes. In an attempt to improve the stability of the fluorophore-conjugated vitamin $\mathrm{C}$, we chose a 6-aminohexyl chain to serve as the linker joining AA and the fluorescent moiety (RB). We reasoned that use of this long spacer arm would keep the bulky fluorophore away from the vicinity of the binding region of the AA skeleton, which would benefit the stability of the ester and facilitate the probe's binding activity. Moreover, sulforhodamine B was used as the fluorescent material because it is negatively charged and it is difficult for it to pass through the cell membrane. ${ }^{21} \mathrm{RB}-\mathrm{A}-\mathrm{Vc}$ was synthesized in two steps.

The first step is to trigger the reaction of the 6-aminoheanoic acid with L-ascorbic acid in sulfuric acid to produce aminoheanoic acid-ascorbic acid ester $(\mathrm{A}-\mathrm{Vc})$. The second step is to conjugate sulforhodamine $\mathrm{B}$ onto the $\mathrm{A}-\mathrm{Vc}$ ester using 4dimethylaminopyridine as a catalyst. The probe $\mathrm{RB}-\mathrm{A}-\mathrm{Vc}$ was purified and identified by liquid chromatography-mass spectrometry and characterized by fluorescent spectrometry (Fig. 2). The introduction of the 6-aminohexyl spacer also introduces an $11 \mathrm{~nm}$ redshift in the emission wave-length of $\mathrm{RB}-\mathrm{A}-\mathrm{Vc}$ compared to RB (Fig. 1B). As we expected, RB-A-Vc shows a very good stability, in which less than $2 \%$ of the original compound is hydrolysed in PBS buffer ( $\mathrm{pH}$ 7.2) within 3 days.

\section{Specificity of RB-A-Vc/SVCT binding}

The target and imaging mechanism is based on the specific interaction between the probe $\mathrm{RB}-\mathrm{A}-\mathrm{Vc}$ and SVCTs that are expressed in cells. When AA is present, however, this process is more likely undergoing a competitive mechanism. We wondered whether exogenetic and endogenetic AA would deteriorate the uptake of probe RB-A-Vc and thus have influence on the SVCT imaging. To this end, the extracted cytoplasmic proteins of the HEK-293T and HepG2 cells were subjected to native-PAGE. The $\mathrm{RB}-\mathrm{A}-\mathrm{Vc}(50 \mu \mathrm{M})$ mixed with different concentrations of ascorbic acid ( 0 to $1 \mathrm{mM}$ ) in the staining buffer was employed to stain the proteins. With the increase of the $\mathrm{AA} / \mathrm{RB}-\mathrm{A}-\mathrm{Vc}$ ratio in the staining buffer, in which AA competes with the RB-A-Vc/SVCT binding, the fluorescence of $\mathrm{RB}-\mathrm{A}-\mathrm{Vc}$ was not reduced compared with the control (AA concentration of 0) (Fig. 3). SVCTs seem to preferentially bind $50 \mu \mathrm{M}$ RB-A-Vc even in the 
a)

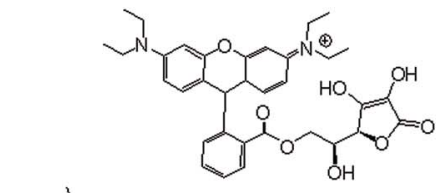

c)

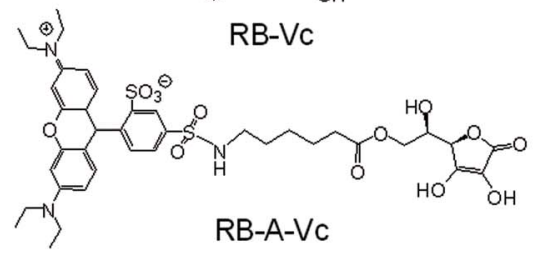

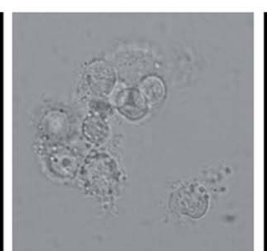

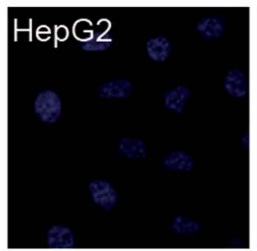

DAPI

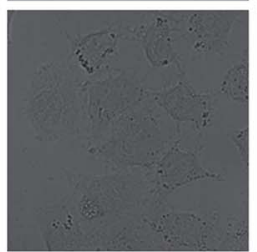

Bright Field
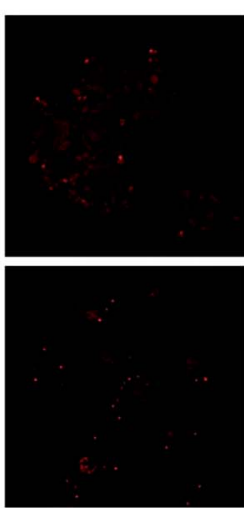

RB-A-VC b)
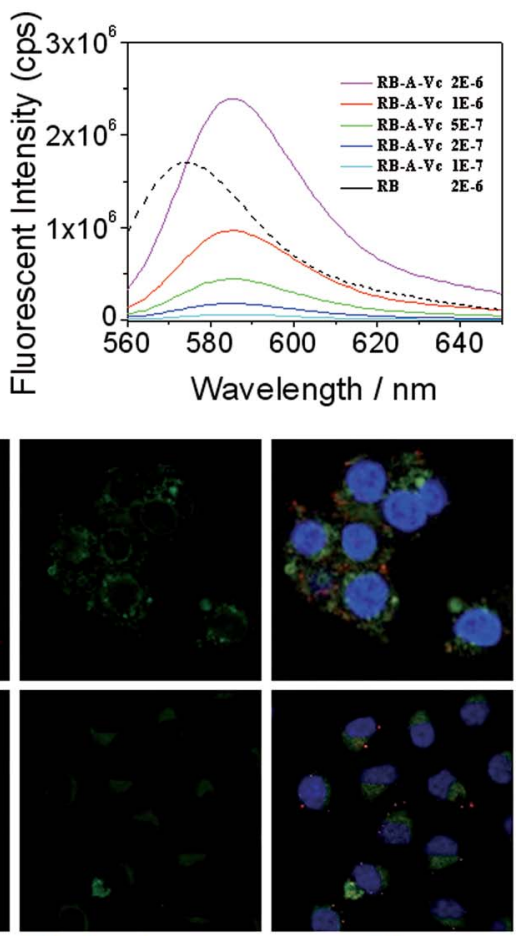

Mito-tracker green

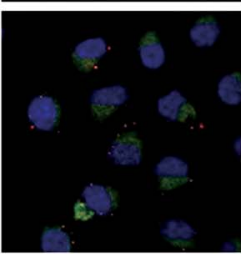

Merge

Fig. 1 (a) Chemical structures of RB-Vc and RB-A-Vc; (b) fluorescence spectra of the RB-A-Vc probe (solid lines) and Rhodamine B (dash line); (c) confocal fluorescent images of HEK-293T and HepG2 cells that were fixed with 4\% paraformaldehyde and permeabilized with $0.1 \%$ Triton X100, stained with RB-A-Vc (red), DAPI (blue) and Mito-tracker green (green). Merged images show the colocalization of mitochondria with SVCT/RB-A-Vc complexes.

presence of a high concentration of AA (1 mM). It seems that no matter how low or high the AA concentration, SVCTs bind with $\mathrm{RB}-\mathrm{A}-\mathrm{Vc}$ preferentially. We explain this by considering that the affinity of RB-A-Vc against SVCTs is higher than that of AA. This observation coincides with the previous findings that $\mathrm{C} 6-\mathrm{OH}$ derived AA esters usually exhibit higher affinity against SVCTs over the endogenous $\mathrm{AA}^{22,23}$ Thus, it is not necessary to be worried about the AA influence on SVCT imaging by competition with RB-A-Vc binding. Moreover, the high affinity leads to a fast binding and slow dissociation of $\mathrm{RB}-\mathrm{A}-\mathrm{Vc} / \mathrm{SVCT}$ complexes, greatly facilitating the fluorescence imaging of the complexes in a relatively stable state.

\section{Fluorescence imaging of SVCTs in the fixed cells}

Prior to the living cell visualization experiments, we first examined the applicability of the synthesized RB-A-Vc for SVCT imaging in the fixed cells. In this experiment, the fixed human

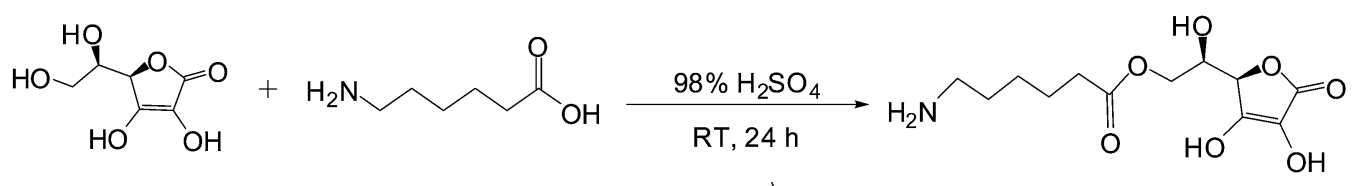

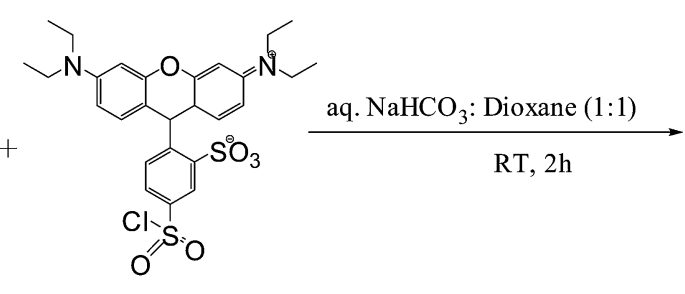<smiles>CCN(CC)C1CCC2C(C1)OC1CC(N(CC)CC)CCC1C2C1CCC(S(=O)(=O)NCCCCCC(=O)OCC(O)C2OC(=O)C(O)C2O)C(=O)O1</smiles>

Scheme 1 Synthesis of sulforhodamine B-aminoheanoic acid-ascorbic acid ester (RB-A-Vc, 2). RB-A-Vc was synthesized in two steps: (1) triggering the reaction of 6 -aminoheanoic acid with $L$-ascorbic acid in sulfuric acid, producing aminoheanoic acid-ascorbic acid ester (A-Vc, 1 ), and (2) conjugating sulforhodamine B onto A-Vc ester using 4-dimethylaminopyridine as the catalyst. 
a)

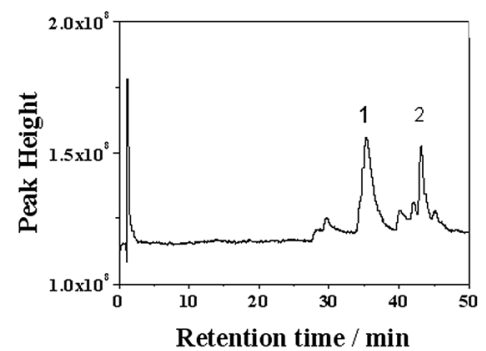

b)

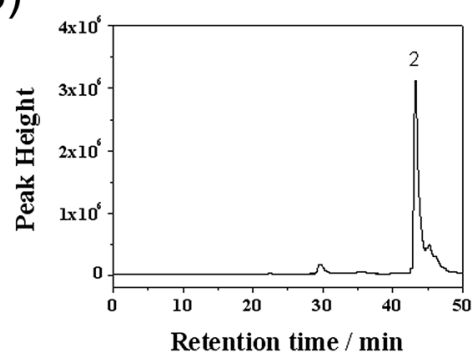

c)

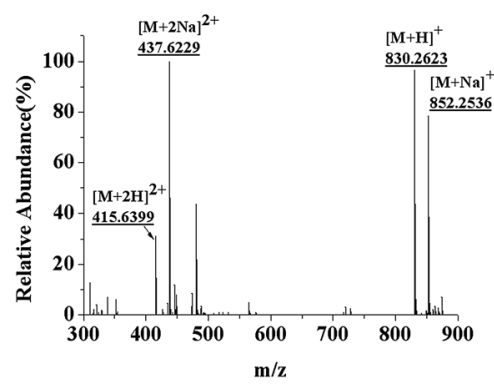

Fig. 2 UHPLC-ESI-MS/MS analysis of RB-A-Vc. (a) TIC spectrum of the crude products of the reaction; 1 is assigned to sulforhodamine $B$ chloride and 2 is assigned to the probe $R B-A-V_{c}$; (b) EIC spectrum of RB-A- $V_{c}$; (c) MS spectrum of RB-A-Vc extracted from TIC, in which [M + $\mathrm{H}]^{+}$of $\mathrm{RB}-\mathrm{A}-\mathrm{Vc}$ is $830.26,[\mathrm{M}+2 \mathrm{H}]^{2+}$ is 415.64 , and $[\mathrm{M}+2 \mathrm{Na}]^{2+}$ is 437.63 . The detection of the expected molecular species indicates that the reaction successfully produced the product.

HEK-293T and hepatoma HepG2 cells, which were treated with $4 \%$ paraformaldehyde and permeabilized with $0.1 \%$ Triton $\mathrm{X}$ 100, were employed for intracellular SVCT monitoring. After treating the fixed cells with $100 \mu \mathrm{M} \mathrm{RB}-\mathrm{A}-\mathrm{Vc}$ we found, by using laser scanning confocal microscopy, that the red fluorescence produced by SVCT2/RB-A-Vc quickly entered into the fixed cells. A mitochondria tracker, Mito-tracker green, was then used to stain the mitochondria in the HEK-293T cells. Interestingly, it was found that the locations of SVCT2/RB-A-Vc represented by the red fluorescence are highly coincident to that of the mitochondria (green fluorescence). This implies that SVCT2 would be primarily located in the mitochondria, which is consistent with the previous studies. ${ }^{24,25}$ Nearly no fluorescence signals could be seen on the membrane of the HEK-293T cell, implying the level of SVCTs in the cell membrane is very low. Confocal colocalization experiments with anti-SVCT2 revealed that most of the SVCT2 immunoreactivity was associated with the mitochondria, and very low immunoreactivity at the plasma membrane (Fig. 4). This confirmed our findings obtained by using RB-A-Vc imaging.

\section{Fluorescence imaging of SVCTs in the living cells}

To gain further insight into the fluorescence sensing and imaging of SVCTs in living cells, we applied the RB-A-Vc for direct staining of the SVCTs in living HEK-293T and HepG2 cells. A fundamental of a SVCTs' function is their potent activation by sodium ions. Uptake of AA by living cells requires the presence of $\mathrm{Na}^{+}$ions, with the cooperation of $\mathrm{Ca}^{2+}$ and $\mathrm{Mg}^{2+}$ ions. ${ }^{26}$ Therefore, in all tests, the probe $\mathrm{RB}-\mathrm{A}-\mathrm{Vc}$ was dissolved in $10 \mathrm{mM}$ HEPES containing $135 \mathrm{mM} \mathrm{NaCl}, 5 \mathrm{mM} \mathrm{KCl}, 2.0 \mathrm{mM}$ $\mathrm{CaCl}_{2}$ and $1.0 \mathrm{mM} \mathrm{MgCl}$. The fluorescence confocal microscopy analysis showed that the $\mathrm{RB}-\mathrm{A}-\mathrm{Vc}$ could quickly enter into the living cells within $30 \mathrm{~min}$. The RB-A-Vc stained living cells were then fixed with paraformaldehyde and permeabilized with Triton X-100, followed by staining steps with DAPI (nuclei, blue) and Mito-tracker green (mitochondria, green). Confocal fluorescence images reveal the successful binding between the RBA-Vc and intracellular SVCT2, showing SVCT2 is mainly localized near the positions of mitochondria (Fig. 5a). We also addressed this issue by performing colocalization experiments using the $\mathrm{RB}-\mathrm{A}-\mathrm{Vc}$ in combination with antibodies against protein markers, in which anti-SVCT2, anti-SVCT1, anti-COXIV for the mitochondria and anti-GLUT1 for the plasma membrane (in the case of HepG2 cells) were employed for subcellular staining. It must be noted that immunofluorescence antibodies including anti-SVCT2 and anti-SVCT1 bind SVCTs in a quite different manner from $\mathrm{AA}$ or $\mathrm{RB}-\mathrm{A}-\mathrm{Vc}$, thereby not interfering with the forming of SVCT/RB-A-Vc complexes. ${ }^{27}$ Confocal colocalization experiments with anti-SVCT2 and anti-organelle a)

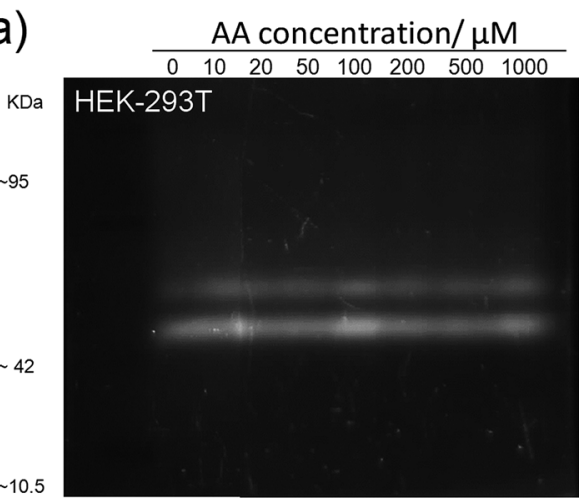

b)

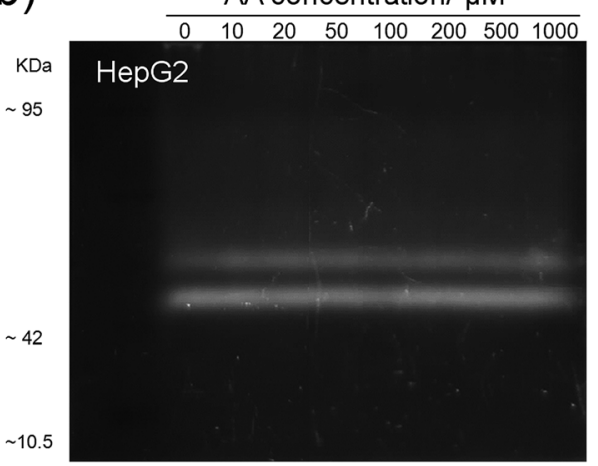

Fig. 3 Native-PAGE of extracted cytoplasmic proteins of HEK-293T and HepG2 cells. SVCTs seem to preferentially bind $50 \mu M$ of RB-A-Vc even in the presence of a high concentration of AA (1 mM). 

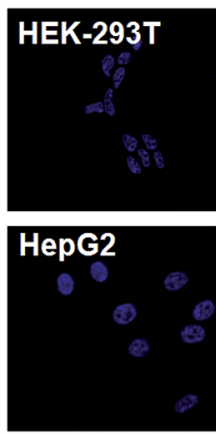

DAPI
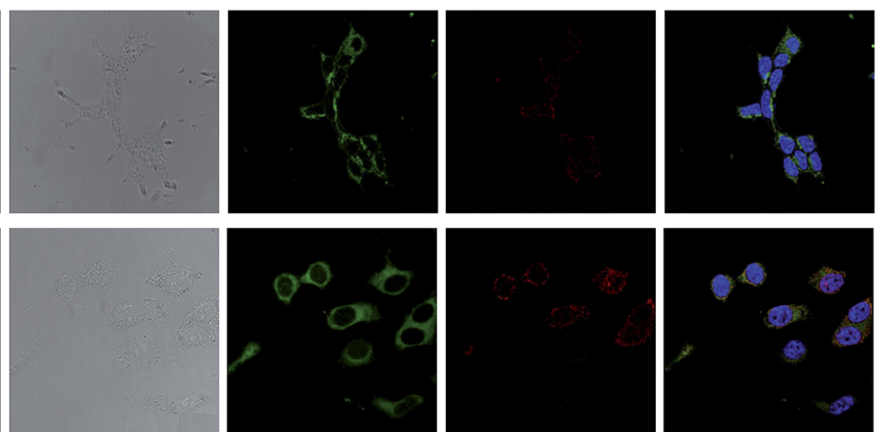

Bright Field

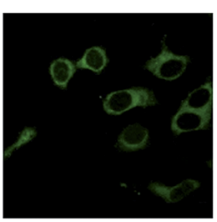

Mito-tracker green

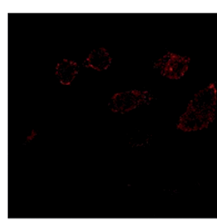

nti-SVCT2

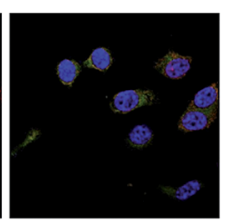

Merge

Fig. 4 Confocal colocalization of anti-SVCT2 (in red) with mitochondria dye Mito-tracker green (in green) in HEK-293T (the upper) and HepG2 cells (the bottom). Cells were fixed with $4 \%$ paraformaldehyde and permeabilized with $0.1 \%$ Triton X-100, incubated with the primary anti-SVCT2 and then the second antibody Cy3-conjugated affiniPure Donkey Anti-Goat IgG $(\mathrm{H}+\mathrm{L})(\mathrm{red})$, stained nuclei with DAPI (blue).

protein markers revealed that most of the SVCT2 immunoreactivity was associated with the mitochondria, with minor colocalization at the endoplasmic reticulum and very low immunoreactivity at the plasma membrane. The transporter SVCT1 is absent from HEK-293T cells. Unlike HEK-293T cells, both SVCT1 and SVCT2 were detected in HepG2 cells. SVCT2 a)

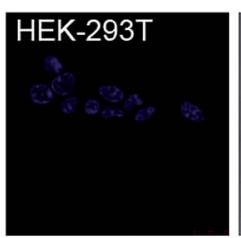

DAPI

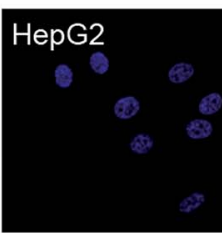

DAPI

b)
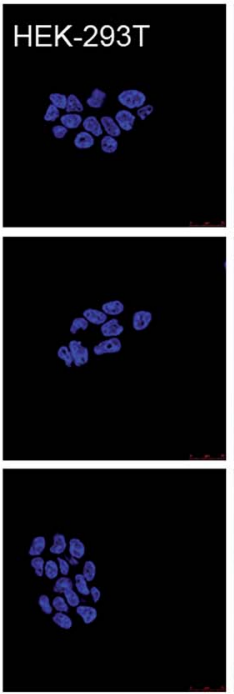

DAPI

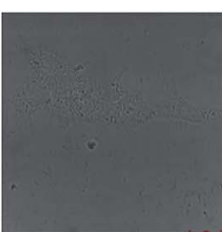

Bright Field

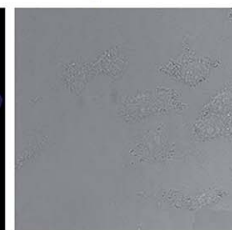

Bright Field

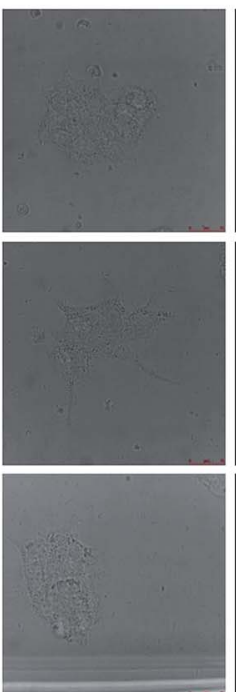

Bright Field
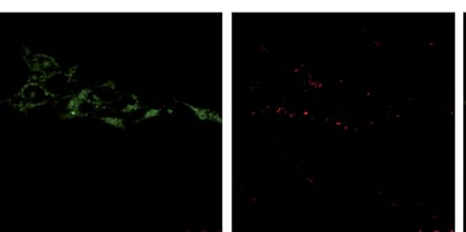

Mito-tracker green
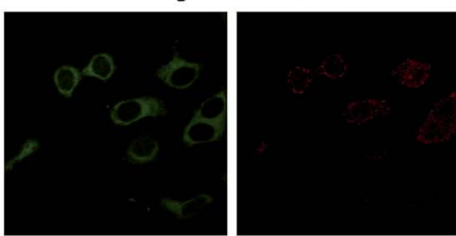

RB-A-VC
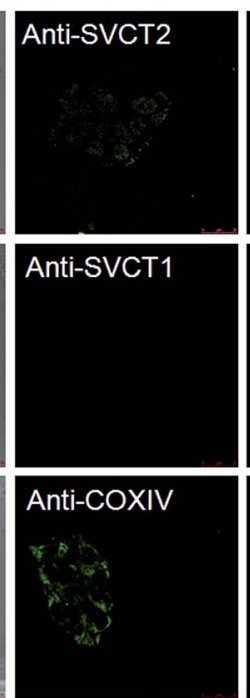

Antibody
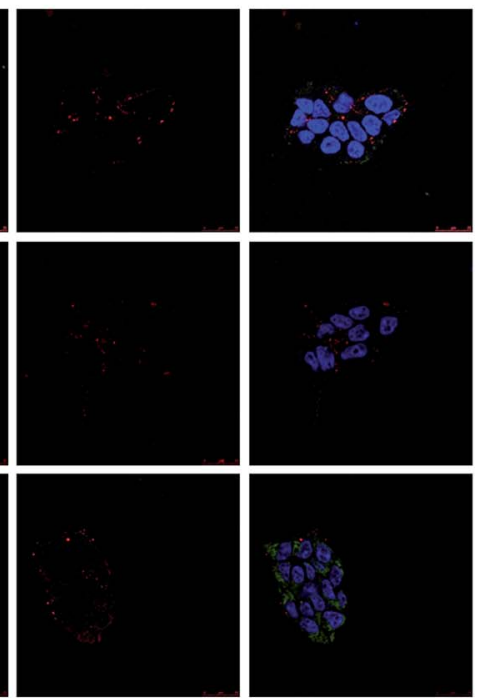

RB-A-Vc

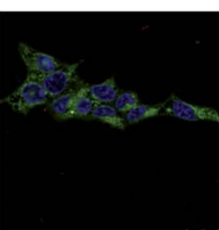

Merge

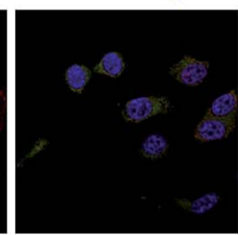

Merge

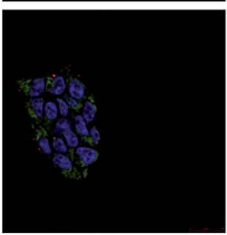

Merge

Fig. 5 (a) Confocal colocalization of SVCT2/RB-A-Vc with Mito-tracker green in living HEK-293T (the upper) and HepG2 (the bottom) cells; (b) confocal colocalization of RB-A-Vc with anti-SVCT2, anti-SVCT1 and anti-COXIV in HEK-293T cells. 
was primarily found at the positions of the mitochondria and slightly observed on the membrane. Meanwhile, a large amount of SVCT1 was observed on the membrane of HepG2 cells. The intracellular distributions of SVCTs depicted by the antibodies were in accordance with that monitored by the $\mathrm{RB}-\mathrm{A}-\mathrm{Vc}$ probe. $\mathrm{RB}-\mathrm{A}-\mathrm{Vc}$ could be used as a living cell fluorescent probe to realize the subcellular localization.

It is noteworthy that the isoelectric point (pI) of the synthesized RB-A-Vc was measured as 5.2. Under the physiological $\mathrm{pH}$ conditions ( $\mathrm{pH} 7.2$ ), RB-A-Vc became negatively-charged and difficult to permeate the cell membrane without the aid of transporters. ${ }^{28}$ To further confirm the RB-A-Vc was transported by SVCTs, we examined the specificity of $\mathrm{RB}-\mathrm{A}-\mathrm{Vc}$ against SVCTs by Western blot assay. The results indicate there is only SVCT2 identified at $\sim 50 \mathrm{kDa}$, but no SVCT1 (at $65 \mathrm{kDa}$ ) was found in HEK-293T cells (Fig. 6a).

\section{Dosage effect of RB-A-Vc on SVCT imaging}

To investigate the dosage effect of the $\mathrm{RB}-\mathrm{A}-\mathrm{Vc}$ on SVCT imaging, different concentrations of $\mathrm{RB}-\mathrm{A}-\mathrm{Vc}$ were added to the cultured living cells. Fluorescence measurements by microplate reader revealed that, in a wide range of $\mathrm{RB}-\mathrm{A}-\mathrm{Vc}$ concentration (from 10 to $200 \mu \mathrm{M}$ ), the fluorescence responses continuously increased both for HEK-293T and HepG2 cells (Fig. 6b). This phenomenon was also observed by confocal fluorescence imaging and flow cytometry analysis (Fig. 6c). From 10 to
$200 \mu \mathrm{M}$ of the RB-A-Vc, the fluorescence responses of the RBA-Vc/SVCT complexes in HEK-293T cells, which were predominately located in cellular plasma near the mitochondria, became brighter. When the RB-A-Vc concentration is higher than $200 \mu \mathrm{M}$ however, redundant fluorescence signals were observed on the cell membrane and in the adjacent areas between the individual cells. Considering the fact that HEK293 T cells do not express high level SVCTs on the membrane, the emerging fluorescences are more likely generated from the non-selective interactions between the probe and biomolecules on the cell surface. To avoid the non-selective interactions, 100 $\mu \mathrm{M}$ of $\mathrm{RB}-\mathrm{A}-\mathrm{Vc}$ was selected for intracellular SVCTs investigation.

\section{Indication of changes in SVCT expression in living cells}

After the validation of the RB-A-Vc's function in SVCT labelling and localization, we considered whether it could indicate the changes of SVCT expression in living cells. For this purpose, we first examined the situation of suppression of SVCT expression. Human HEK-293T cells were transfected with SVCT2 siRNA or a negative control (NC) siRNA within $72 \mathrm{~h}$, and then the SVCT2 content was examined by Western blot. To the siRNA treated $293 \mathrm{~T}$ cells, $100 \mu \mathrm{M}$ of the RB-A-Vc was added and then visualized by confocal fluorescent imaging. HEK-293T cells showed decreased fluorescence intensity after the SVCT2 siRNA treatment (Fig. 7a). Fluorescence analysis and Western blotting a)

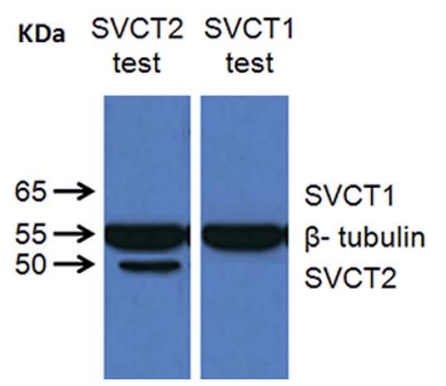

c)

b)

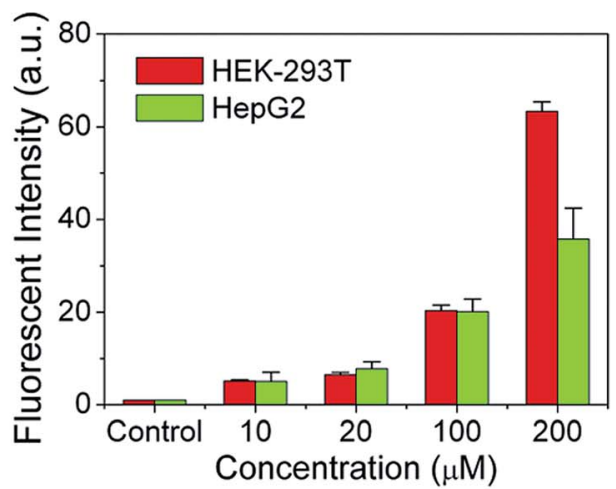

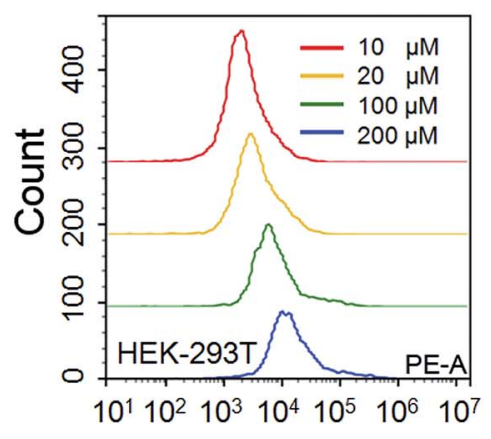

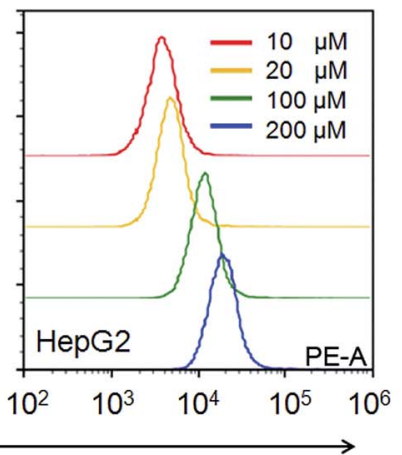

Fluorescence of SVCTs/RB-A-Vc

Fig. 6 (a) Western blot of SVCT2 and SVCT1 expression in HEK-293T cells, (b) the dosage effect of RB-A-Vc concentration on fluorescence responses by microplate reader; (c) the dosage effect measured by flow cytometry analysis. 
a)

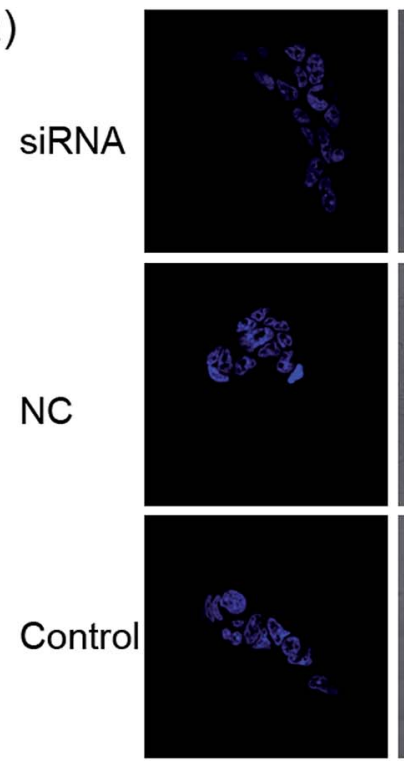

DAPI
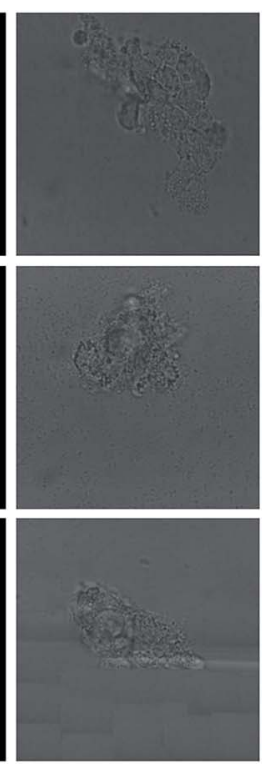

Bright field
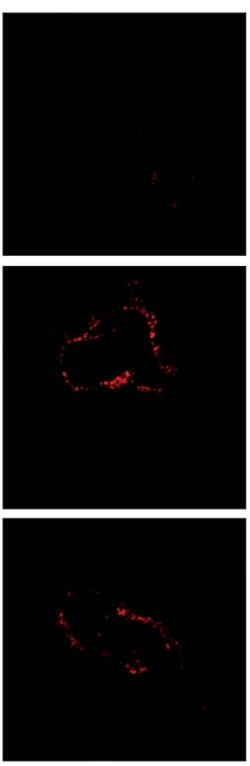

RB-A-Vc
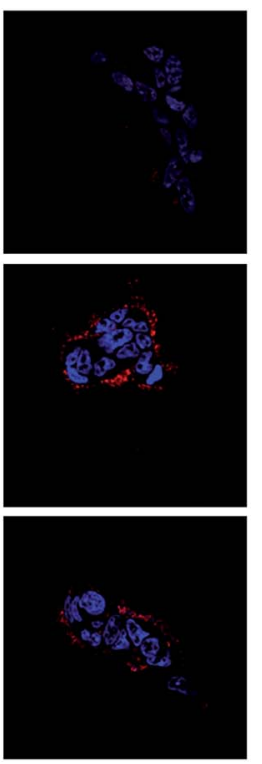

Merge b)

\section{SVCT2}

$\beta$-tubulin
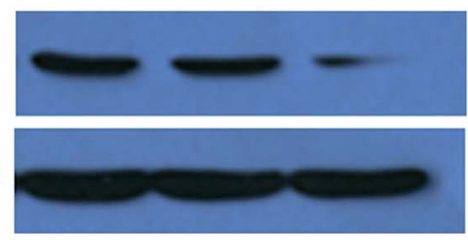

Ctrl NC siRNA c)

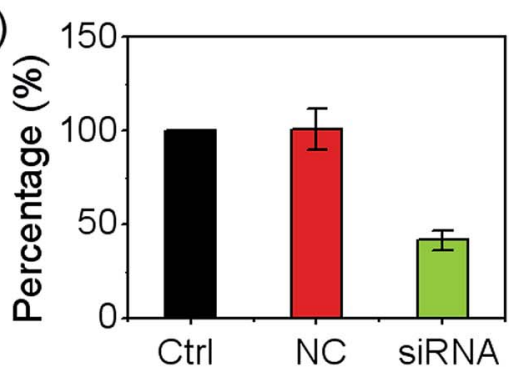

Fig. 7 Use of SVCT2 siRNA for silencing SVCT2 expression produced major decreases in mitochondria. (a) Immunoblotting revealed SVCT2 protein expression decreased by approximately 75\%; (b) Western blot and (c) fluorescence analysis of SVCT2 expression in SVCT2 siRNA treated HEK-293T cells.

revealed SVCT2 expression decreased by approximately $60 \%$ (Fig. 7b and c). FACS analysis (flow cytometry) also substantiated the decrease in fluorescence of the RB-A-Vc after transfection of SVCT2 siRNA. This implies that the RB-A-Vc can indicate the down-regulation of SVCT2 expression in living cells.

We then validated the function of the $\mathrm{RB}-\mathrm{A}-\mathrm{Vc}$ probe by studying the promotion of SVCT expression in living cells.

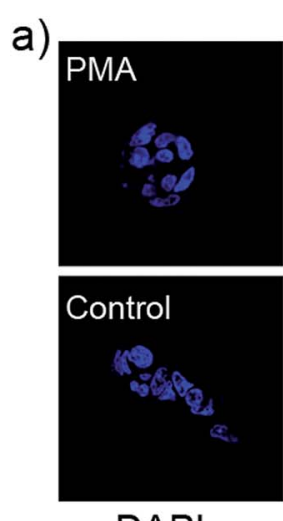

DAPI
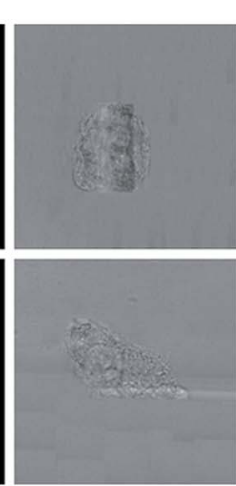

Bright field
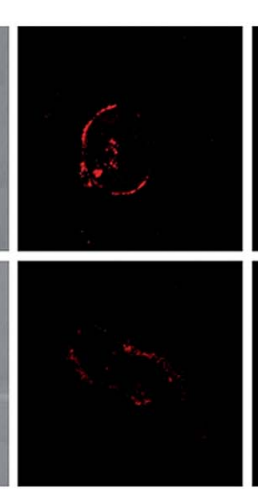

RB-A-Vc

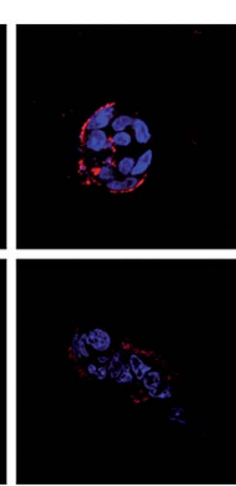

Merge

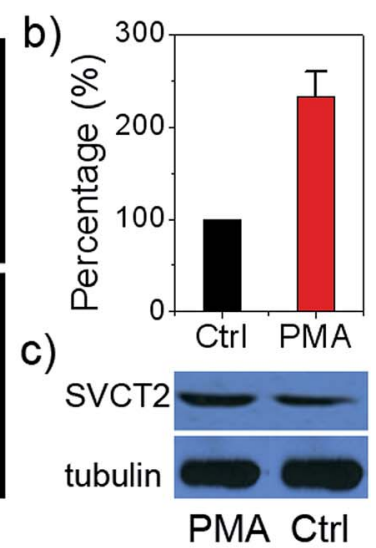

Fig. 8 PMA induces an up-regulated SVCT2 expression in HEK-293T cells. (a) RB-A-Vc visualizes the PMA-induced up-regulation of SVCT2 expression; (b) fluorescence analysis and (c) Western blot of SVCT2 expression in PMA treated HEK-293T cells. 


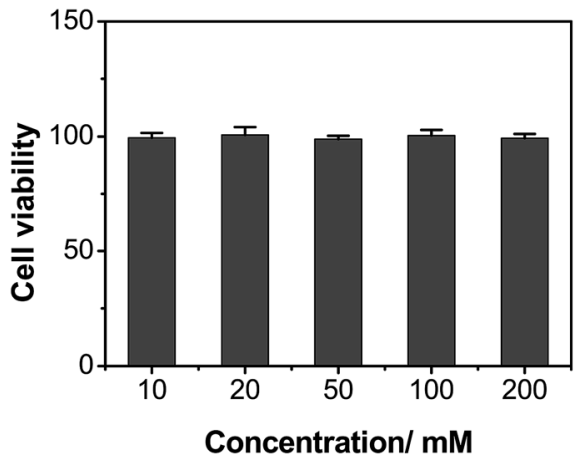

Fig. 9 No observable cytotoxicity of RB-A-Vc $(10-200 \mathrm{mM})$ to the HepG2 cells (MTS assay).

Phorbol 12-myristate 13-acetate (PMA) is a nonselective protein kinase-C activator, and it was reported to cause a large increase in SVCT2 expression..$^{\mathbf{2 2 , 2 9 , 3 0}}$ In this experiment, we added $100 \mathrm{nM}$ PMA to HEK-293T cells to induce an up-regulated SVCT expression, and then extracted the cytoplasmic proteins to conduct a Western blot experiment. After exposure with PMA, the expression of SVCT2 greatly increased. Compared with the control, the intensity of fluorescence of the RB-A-Vc increased obviously after adding 100 nM PMA. Fluorescence analysis and Western blotting revealed up-regulated SVCT2 protein expression by approximately $220 \%$ (Fig. 8). This result suggests that the $\mathrm{RB}-\mathrm{A}-\mathrm{Vc}$ could indicate not only the down-regulation but also the up-regulation of SVCT2 expression. Together, these results support the notion that $\mathrm{RB}-\mathrm{A}-\mathrm{Vc}$ is a potent fluorescent probe to indicate changes of SVCTs which are responsible for the transportation of ascorbic acid into cells.

\section{Cytotoxicity of probe RB-A-Vc}

Moreover, the cytotoxicity of probe RB-A-Vc to HepG2 cells was measured by using a water soluble tetrazolium salt (3-(4,5dimethylthiazol-2-yl)-5-(3-carboxymethoxyphenyl)-2-(4-sulfophenyl)- $2 H$-tetrazolium) (MTS) assay. As shown in Fig. 9, the cellular viability in the presence of either $\mathrm{RB}-\mathrm{A}-\mathrm{Vc}$ or sulforhodamin B chlorine was estimated to be greater than $98 \%$ after $24 \mathrm{~h}$ with an increasing concentration of dye. Hence, the RB-AVc could be regarded to be low in cytotoxicity and suitable for living cell imaging.

\section{Conclusions}

In summary, we have presented the synthesis and application of $\mathrm{RB}-\mathrm{A}-\mathrm{Vc}$, a new small molecule fluorescent probe for selective fluorescence imaging of SVCTs in living cells. This probe features visible excitation and emission profiles, ease of entering into cells, high selectivity for SVCTs, and can monitor up-regulation or down-regulation of SVCT expression in living cells. We emphasize this small-molecule probe is suitable for subcellular localization of SVCTs in living cells. This study provides a potent tool for simultaneously monitoring the level and distribution of intracellular SVCTs, which is probably more useful for evaluating the changes induced by exoteric stimulations. We propose that this probe for SVCT imaging and the corresponding method can be extended to the imaging of other cell lines, tissues, and species.

\section{Acknowledgements}

The work was financially supported by grants from the National Basic Research Program of China (No. 2014CB932003), the Strategic Priority Research Program of the CAS (No. XDB14030300), the National Natural Science Foundation of China (No. 21375142 and 21300076), and the special fund for public benefit from Ministry of Environmental Protection of China (No. 201309045).

\section{Notes and references}

1 F. E. Harrison and J. M. May, Free Radical Biol. Med., 2009, 45, 719.

2 I. Savini, A. Rossi, C. Pierro, L. Avigliano and M. V. Catani, Amino Acids, 2008, 34, 347.

3 M. Hediger, Nat. Med., 2002, 8, 445.

4 J. M. May, Br. J. Pharmacol., 2011, 164, 1793.

5 J. M. May, Z. C. Qu and S. Mendiratta, Arch. Biochem. Biophys., 1998, 349, 281.

6 S. B. Nimse and D. Pal, RSC Adv., 2015, 5, 27986.

7 H. Tsukaguchi, T. Tokui, B. Mackenzie, U. Berger, X. Chen, Y. Wang, R. Brubaker and M. Hediger, Nature, 1999, 399, 70.

8 F. Nualart, L. Mack, A. García, P. Cisternas, E. R. Bongarzone, M. Heitzer, N. Jara, F. Martínez, L. Ferrada, F. Espinoza, V. Baeza and K. Salazar, J. Stem Cell Res. Ther., 2014, 4, 209.

9 F. E. Harrison and J. M. May, Free Radical Biol. Med., 2009, 46, 719.

10 F. E. Harrison, S. M. Dawes, M. E. Meredith, V. R. Babaev, L. Li and J. M. May, Free Radical Biol. Med., 2010, 49, 821.

11 S. Sotiriou, S. Gispert, J. Cheng, Y. H. Wang, A. Chen, S. Hoogstraten-Miller, G. F. Miller, O. Kwon, M. Levine, S. H. Guttentag and R. L. Nussbaum, Nat. Med., 2002, 8, 514.

12 A. I. Acuña, M. Esparza, C. Kramm, F. A. Beltrán, A. V. Parra, C. Cepeda, C. A. Toro, R. L. Vidal, C. Hetz, I. I. Concha, S. Brauchi, M. S. Levine and M. A. Castro, Nat. Commun., 2013, 4, 2917.

13 H. R. Harris, N. Orsini and A. Wolk, Eur. J. Cancer, 2014, 50, 1223.

14 V. Khurana, D. Kwatra, D. Pal and A. K. Mitra, Int. J. Pharm., 2014, 474, 14.

15 X. Xu, E. Yu, L. Liu, W. Zhang, X. Wei, X. Gao, N. Song and C. Fu, Eur. J. Cancer Prev., 2013, 22, 529.

16 S. Huang, F. Zhu, Q. Xiao, W. Su, J. Sheng, C. Huang and B. $\mathrm{Hu}, R S C A d v ., 2014,4,46751$.

17 J. Kim, T. Kino, H. Kato, F. Yamamoto, K. Sano, T. Mukai and M. Maeda, Chem. Pharm. Bull., 2012, 60, 235.

18 G. H. Mun, M. J. Kim, J. H. Lee, H. J. Kim, Y. H. Chung, Y. B. Chung, J. S. Kang, Y. I. Hwang, S. H. Oh, J. G. Kim, D. H. Hwang, D. H. Shin and W. J. Lee, J. Neurosci. Res., 2006, 83, 919.

19 M. E. Meredith, F. E. Harrison and J. M. May, Biochem. Biophys. Res. Commun., 2011, 414, 737. 
20 L. Mardones, F. A. Zúñiga, M. Villagrán, K. Sotomayor, P. Mendoza, D. Escobar, M. González, V. Ormazabal, M. Maldonado, G. Oñate, C. Angulo, I. I. Concha, A. M. Reyes, J. G. Cárcamo, V. Barra, J. C. Vera and C. I. Rivas, Free Radical Biol. Med., 2012, 52, 1874.

21 O. Boussif, F. Lezoualc'h, M. A. Zanta, M. D. Mergny, D. Scherman, B. Demeneix and J. P. Behr, Proc. Natl. Acad. Sci. U. S. A., 1995, 92, 7297.

22 S. Manfredini, B. Pavan, S. Vertuani, M. Scaglianti, D. Compagnone, C. Biondi, A. Scatturin, S. Tanganelli, L. Ferraro, P. Prasad and A. Dalpiaz, J. Med. Chem., 2002, 45, 559.

23 A. Dalpiaz, B. Pavan, M. Scaglianti, F. Vitali, F. Bortolotti, C. Biondi, A. Scatturin, S. Tanganelli, L. Ferraro, P. Prasad and S. Manfredini, J. Pharm. Sci., 2004, 93, 78.

24 C. Muñoz-Montesino, F. J. Roa, E. Peña, M. González, K. Sotomayor, E. Inostroza, C. A. Muñoz, I. González,
M. Maldonado, C. Soliz, A. M. Reyes, J. C. Vera and C. I. Rivas, Free Radical Biol. Med., 2014, 70, 241.

25 M. Burzle and M. A. Hediger, Curr. Top. Membr., 2012, 70, 357.

26 A. Godoy, V. Ormazabal, G. Moraga-Cid, F. A. Zúñiga, P. Sotomayor, V. Barra, O. Vasquez, V. Montecinos, L. Mardones, C. Guzmán, M. Villagrán, L. G. Aguayo, S. A. Oñate, A. M. Reyes, J. G. Cárcamo, C. I. Rivas and J. C. Vera, J. Biol. Chem., 2007, 282, 615.

27 S. Varma, K. Sobey, C. E. Campbell and S. M. Kuo, Biochemistry, 2009, 48, 2969.

28 S. Luo, Z. Wang, V. Kansara, D. Pal and A. Mitra, Int. J. Pharm., 2008, 358, 168.

29 H. Qiao and J. M. May, Free Radical Biol. Med., 2009, 46, 1221. 30 V. Ulloa, M. García-Robles, F. Martínez, K. Salazar, K. Reinicke, F. Pérez, D. F. Godoy, A. S. Godoy and F. Nualart, J. Neurochem., 2013, 127, 403. 